\title{
Special Agents Hunting Down Women Silent Killer: The Emerging Role of the p38 $\alpha$ Kinase
}

\author{
Valentina Grossi and Cristiano Simone \\ Laboratory of Signal-dependent Transcription, Department of Translational Pharmacology (DTP), Consorzio Mario NegriSud 66030, \\ Santa Maria Imbaro, Italy \\ Correspondence should be addressed to Cristiano Simone, simone@negrisud.it
}

Received 20 September 2011; Revised 21 December 2011; Accepted 29 December 2011

Academic Editor: Ritu Salani

Copyright ( $\odot 2012$ V. Grossi and C. Simone. This is an open access article distributed under the Creative Commons Attribution License, which permits unrestricted use, distribution, and reproduction in any medium, provided the original work is properly cited.

\begin{abstract}
Ovarian cancer is sensitive to chemotherapy with platinum compounds; however, the therapy success rate is significantly lowered by a high incidence of recurrence and by the acquisition of drug resistance. These negative outcomes mainly depend on altered apoptotic and drug resistance pathways, determining the need for the design of new therapeutic strategies to improve patient survival. This challenge has become even more critical because it has been recognized that hindering uncontrolled cell growth is not sufficient as the only curative approach. In fact, while current therapies are mostly conceived to impair survival of highly proliferating cells, several lines of research are now focusing on cancer-specific features to specifically target malignant cells with the aim of avoiding drug resistance and reducing adverse effects. Recently, great interest has been generated by the identification of metabolic reprogramming mechanisms occurring in cancer cells, such as the increase in glycolysis levels. In this light, pharmacologic manipulation of relevant pathways involved in cancer-specific metabolism and drug resistance could prove an effective approach to treat ovarian cancer patients.
\end{abstract}

\section{Introduction}

Ovarian cancer has historically been called the "silent killer," even if around $80 \%$ of patients do actually have symptoms. Indeed, only $20 \%$ of ovarian cancers are currently diagnosed while still limited to the ovaries, when up to $90 \%$ of patients can be cured using available therapies. Its poor prognosis is related to late diagnosis, which usually occurs at advanced stages, and to acquisition of chemoresistance [1]. To date, more than 30 oncogenes and tumor suppressor genes have been identified that are involved in ovarian oncogenesis inducing modifications in proliferation, apoptosis, anoikis, motility, adhesion, and invasion [2].

\section{Genetic Alterations in Ovarian Cancer}

Although ovarian cancer risk is, at least in part, influenced by hormonal, environmental, and racial factors, a major role is played by genetic factors. Indeed, a key advance in the study of ovarian cancer etiology has been the identification of mutations in the BRCA genes. BRCA1 and BRCA2 genes act as tumor suppressor genes and, when mutated, are associated with the accumulation of chromosomal abnormalities and thus with a higher risk of developing cancer. Inheritance of mutations in BRCA genes is associated with a $27 \%$ to $44 \%$ lifetime risk of ovarian cancer. A higher incidence of carcinomas of the ovary has also been detected in families affected by the HNPCC syndrome (hereditary nonpolyposis colorectal cancer) [3], which is caused by mutations in DNA mismatch repair genes. HNPCC carriers account for approximately $1 \%$ of ovarian cancer patients, and their estimated lifetime risk of ovarian cancer is $9 \%$ to $12 \%$ [4].

Mutations in BRAF, KRAS, and erbB2 oncogenes and in the tumor suppressor PTEN have been found in a large subset of ovarian cancers $[5,6]$. The inactivation of PTEN and an activating mutation of KRAS are sufficient to induce ovarian endometrioid carcinoma in a mouse model [7]. Furthermore, mutations of beta-catenin have been detected both in ovarian carcinomas and in their precursor lesions [8]. Indeed, inactivation of the Wnt/beta-catenin and 
the PI3K/PTEN pathways has been shown to induce the development of endometrioid carcinoma in an engineered mouse model [9]. The small G-protein RAB25, which regulates motility, aggressiveness, apoptosis, and autophagy and mediates survival in response to stress, has also been found upregulated in the majority of ovarian cancers [10].

The Aurora-A kinase (Aurora-A) is associated with tumor initiation and progression and is overexpressed in various malignancies. Inhibition of Aurora-A induces cell cycle arrest and decreases proliferation of epithelial ovarian cancer stem cells, which represent the chemoresistant population and act as a source of recurrence [11]. All of these and several other amplified oncogenes are potential targets for ovarian cancer therapy.

2.1. Chromatin Remodeling and Ovarian Cancer. Molecular genetic changes in chromatin remodeling genes have been identified as a new mechanism in cancer pathogenesis. ARID1A (BAF250a), which promotes the formation of SWI/ SNF chromatin remodeling complexes containing BRG1 or BRM, has emerged as a candidate tumor suppressor gene based on its frequent mutations in gynecological cancers. $46 \%-57 \%$ of ovarian clear cell carcinomas, $40 \%$ of uterine endometrioid carcinomas, and $30 \%$ of ovarian endometrioid carcinomas display somatic sequence mutations in ARID1A [12-14]. Guan and colleagues recently reported that restoring wild-type ARID1A expression in ovarian cancer cells that harbor ARID1A mutations is sufficient to suppress cell proliferation and tumor growth in mice. Moreover, they showed that ARID1A/BRG1 complexes directly interact with p53 and that mutations in the ARID1A and TP53 genes were mutually exclusive in tumor specimens. The regulation of p53-related genes by ARID1A raises the possibility that ARID1A cooperates at the molecular level with p53 to inhibit tumor growth. In non-transformed cells, ARID1A and p53 act as a pair of gatekeepers that prevent tumorigenesis by transcriptional activation of tumor-inhibiting downstream genes, such as CDKN1A and SMAD3. The authors found that all tumors with mutated ARID1A contained wildtype TP53 and tumors with mutated TP53 harbored wildtype ARID1A. Mutations in either ARID1A or TP53 were sufficient to inactivate the ARID1A/BRG1/p53 complex and silence transcription of CDKN1A and SMAD3. This recent study suggests a close collaboration between genetic and epigenetic alterations in cancer pathogenesis [15].

2.2. Imprinting and Ovarian Cancer. Genomic imprinting is a molecular mechanism that plays an important role in development, growth, and cell differentiation in mammals. However, only 74 genes have been identified as imprinted among the over 30,000 that can be expressed in human cells. Several of these imprinted genes have been implicated in human oncogenesis. Indeed, while functional inactivation of non-imprinted genes usually requires two genetic alterations, loss of function of imprinted genes may occur following a single genetic or epigenetic event (including loss of heterozygosity (LOH), hypermethylation, and altered transcriptional regulation) occurring on the single functional allele. Moreover, in the case of ovarian oncogenesis, spontaneous mutations may occur during the proliferation of ovarian epithelium to repair ovulatory defects. In this light, downregulation of the imprinted growth-inhibitory genes Aplasia Ras homologue member I (ARHI) and paternally expressed 3 (PEG3) may be particularly important in the pathogenesis of ovarian cancer [16].

ARHI, also known as DIRAS3, is a maternally imprinted tumor suppressor gene encoding a $26 \mathrm{kDa}$ GTPase with $55 \%-62 \%$ homology to Ras and Rap, which inhibits cancer cell growth, motility, and invasion. It is expressed by ovarian epithelial cells and is lost or markedly downregulated in $60 \%-70 \%$ of ovarian cancers [17-19]. Loss of ARHI expression is associated with tumor progression and poor prognosis, while its re-expression in cancer cells inhibits signaling through the Ras/MAPK pathway, induces p21WAF1/CIP1, and downregulates cyclin D1 [19]. Besides, Lu et al. [16] demonstrated that ARHI re-expression causes autophagic death of ovarian cancer cells in culture and participates directly in autophagosome formation by upregulating the ATG4 enzyme that processes the microtubule-associated protein LC3I to LC3II. Autophagy is a process of "self-eating" that involves enzymatic digestion and recycling of cellular constituents in response to stress. While it can contribute to cancer cell death in response to chemotherapeutic agents [20], its role in oncogenesis remains ambiguous as it may also permit survival of cancer cells in response to environmental stress or cytotoxic drugs [21-23]. Indeed, induction of ARHI in xenografts does not kill ovarian cancer cells but instead induces tumor dormancy [24], and its subsequent downregulation rapidly resumes cancer growth.

PEG3 is an imprinted gene encoding a $140 \mathrm{kD}$ Kruppeltype $(\mathrm{C} 2 \mathrm{H} 2)$ zinc-finger protein that plays an important role in the p53/c-myc-mediated apoptotic pathway. It is significantly downregulated in the majority of ovarian cancers due to promoter hypermethylation and $\mathrm{LOH}$, and its re-expression markedly inhibits ovarian cancer growth. Of note, a high degree of correlation has been found between ARHI and PEG3 in terms of mRNA levels and promoter methylation [25].

\section{Current Therapies and New Therapeutic Targets}

The platinum compounds cisplatin and carboplatin are the most effective chemotherapy agents currently used in ovarian cancer. The antitumor activity of cisplatin (cis-diamminedichloroplatinum (II)) was discovered by Rosenberg and colleagues in 1961 [26]. Cisplatin has been the most active drug used for the treatment of ovarian cancer for the last 4 decades, and response to cisplatin is considered a prognostic factor for patients with ovarian cancer [27]. A high percentage of women with ovarian cancer respond to frontline platinum combination chemotherapy, but in most of them the disease will become resistant to cisplatin, ultimately leading to death [27]. Thus, methods of preventing resistance to cisplatin could prove very useful against ovarian cancer. 
The classical therapeutic sequence combines maximal debulking surgery followed by adjuvant platinum- and paclitaxel-based chemotherapy [28,29]. Unfortunately, $20 \%$ of patients do not respond to chemotherapy and recurrent disease occur in $>50 \%$ of those who initially achieve complete remission, with a 5-year overall survival of only $30 \%-$ $40 \%$ for all stages [30].

New therapeutic approaches based on targeted biologic agents have generated great interest and are currently being investigated in several clinical trials focused on treatments for recurrent ovarian cancer (Figure 1). As is the case for other cancers, angiogenesis is a key process implicated in the metastatization of ovarian cancer. Several growth factors, including vascular endothelial growth factor A (VEGFA), lysophosphatidic acid (LPA), interleukin 6 (IL6), interleukin 8 (IL8), and fibroblast growth factor 1 (FGF1) and 2 (FGF2) are involved in this process $[31,32]$. To date, agents that target the VEGF pathway have proven the most effective against the disease.

VEGFA activity has been inhibited by various mechanisms. Bevacizumab, a VEGFA-specific antibody, induced an objective response rate in $16 \%$ of patients with recurrent ovarian cancer and stabilized disease for 5.5 months in $50 \%$ of patients [33], while improved response rates have been observed in platinum-resistant disease when it was used in combination with cytotoxic chemotherapy [34]. The VEGF Trap is based on a different approach [35]: it is a fusion protein that acts as a soluble VEGF receptor and binds with high affinity to VEGF. Several small molecule inhibitors have been used in ovarian cancer to target VEGF and other pathways. Sorafenib, an oral multikinase inhibitor with activity against Raf and other receptor kinases (including the VEGF receptor (VEGFR), the platelet-derived growth factor receptor (PDGFR), and c-Kit) may have antiangiogenic effects through inhibition of VEGFR. This inhibitor has also been used with promising results in combination with bevacizumab and in combination with chemotherapy, both in recurrent disease and as initial therapy in newly diagnosed patients. Sunitinib is an oral agent that inhibits a number of receptor tyrosine kinases implicated in epithelial ovarian cancer (EOC) growth and metastasis, including VEGFR and PDGFR. It has been assessed in phase II studies for the treatment of advanced or metastatic recurrent EOC [36]. Cediranib (AZD2171) is an oral tyrosine kinase inhibitor with selective activity against VEGFR1, VEGFR2, VEGFR3, and c-Kit. Recent clinical trials showed that cediranib has anticancer activity in recurrent EOC [37]. Pazopanib is an oral angiogenesis inhibitor targeting VEGFR, PDGFR, and c-Kit, which is currently being tested in clinical trials on ovarian cancer.

The epidermal growth factor receptor (EGFR) family is commonly overexpressed in ovarian cancer and has been associated with a negative prognosis; however, limited efficacy has been observed with molecules targeted to the EGFR pathway. Gefitinib and erlotinib, which are inhibitors of EGFR, stabilized disease in $11 \%-44 \%$ of patients with ovarian cancer but produced objective regression in only $4 \%-$ $6 \%$ of cases $[38,39]$. The effect of EGFR inhibitors might be reduced by activation of the RAS-MAPK signalling pathway, as happens in colorectal cancers [40]. ErbB2 (also known as HER2) expression in ovarian cancer is associated with advanced stage, higher recurrence frequency, shorter survival time, and lower sensitivity to platinum-based chemotherapy. Trastuzumab and pertuzumab are humanized antibodies targeted against HER2, which act through different mechanisms [41, 42]. In phase II monotherapy clinical studies, trastuzumab has shown activity in certain ovarian cancers overexpressing HER2, while pertuzumab is currently undergoing ovarian cancer trials in combination with cytotoxic agents including gemcitabine [43] and carboplatin [44].

The estrogen receptor $\alpha(\mathrm{ER} \alpha)$ has also been targeted for the treatment of ovarian cancer. Phase II trials of aromatase inhibitors (AIs) have shown modest response but rather better disease stabilization rates, especially when patients are selected on the basis of ER $\alpha$ expression [45].

Activation of the PI3K pathway, which occurs in approximately $70 \%$ of ovarian cancers, is associated with resistance to cytotoxic chemotherapy. Inhibitors of PI3K and Akt prevent the growth of ovarian cancer xenografts and potentiate the cytotoxic effects of paclitaxel and cisplatin [46]. Perifosine is an alkylphospholipid compound that inhibits Akt and is currently being tested in combination with docetaxel. Development of more specific Akt inhibitors is currently underway and PI3K inhibitors are entering phase I-II trials [47].

Overexpression of IL6 has been detected in the majority of ovarian cancers. It induces a signaling pathway that ultimately stimulates proliferation, inhibits apoptosis, and promotes angiogenesis. Antibodies against IL6 and inhibitors of proteins involved in its pathway, such as JAK2 and STAT3, are currently in development for use in ovarian cancer [48].

Upregulation of the LPA receptors LPAR2 and LPAR3 has been described during the malignant transformation of ovarian surface epithelial cells. An approach targeting this pathway in ovarian cancer cells through antibodies capable of neutralizing LPA and through inhibitors of LPA receptors is currently being studied [49].

Constitutive activation of the NFkB transcription factor has been observed in the majority of ovarian cancers [50, 51]. Activated NFKB induces upregulation of anti-apoptotic genes, growth regulatory cytokines (IL6 or growth regulated $\alpha(\mathrm{Gro})$ ), and angiogenic factors (IL8) [52]. A clinical trial is currently underway to study the efficacy of liposomal adenoviral E1A, which interferes with NFKB signaling, in combination with paclitaxel in patients with recurrent ovarian cancer.

The use of poly(ADP-ribose) polymerase (PARP) inhibitors in ovarian cancer is being evaluated in various preclinical, and clinical studies. By interfering with PARP singlestrand DNA repair activity, this strategy is aimed at increasing the cytotoxicity associated with DNA damage induced by chemotherapy and takes advantage of the fact that loss of function of BRCA genes, which are also involved in DNA strand breaks repair, is a common feature of this type of cancer [53]. Response to treatment has been observed in 46\% of ovarian cancer patients with a BRCA mutation administered with the oral PARP inhibitor AZD2281 (Olaparib) [54]. Moreover, several clinical trials are studying the efficacy of PARP inhibitors in combination with cytotoxic compounds 


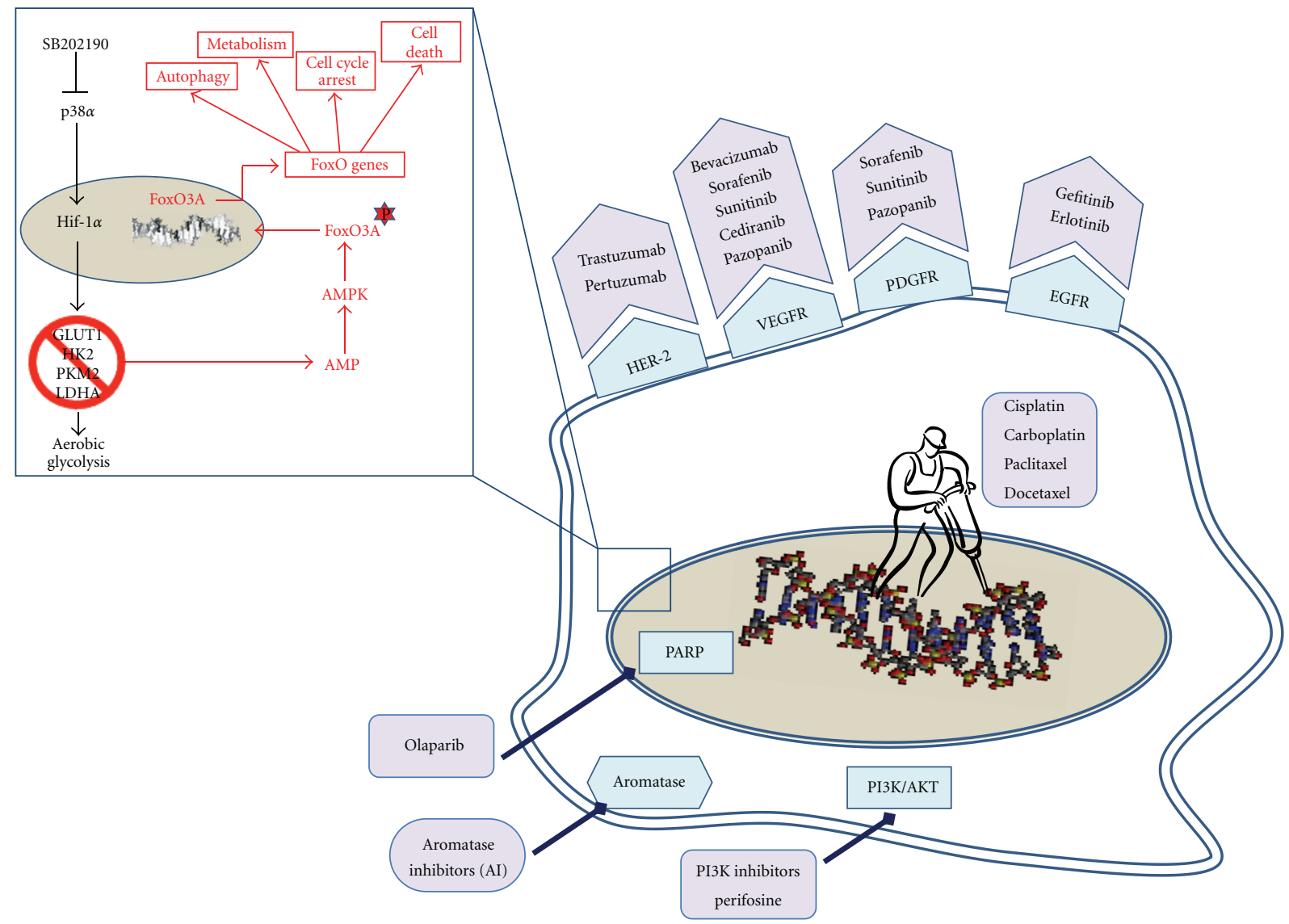

FIGURE 1

including monofunctional alkylating agents, topoisomeraseI poisons and DNA-crosslinking agents [55].

\section{4. p38 $\alpha$ and Ovarian Cancer Cell Survival}

The high rate of drug resistance acquisition observed in ovarian cancer patients has led to a recent shift in the design of therapeutic strategies: pathways involved in drug resistance are being investigated in depth in order to identify new putative targets, and the potential to manipulate cancer-specific features is being evaluated with the aim of specifically targeting tumor cells in order to reduce adverse effects (Figure 1). As for this second aspect, major attention has been focused on the metabolic reprogramming occurring in cancer cells, which display increased levels of glycolysis compared with their normal counterparts. Indeed, conventional therapies, such as chemotherapy and radiation, produce heavy adverse effects because they are mainly designed to affect survival of highly proliferating cells and thus also damage healthy tissues characterized by a high cellular turnover. In recent years, the observation made in the 1920s by Nobel Prize winner Otto Warburg that tumor cells produce $50 \%$ of their adenosine triphosphate through the glycolytic flux versus the $10 \%$ observed in normal cells - the so-called Warburg effect-is being revalorized and is now considered a promising target for new therapeutic approaches [56]. This phenomenon is already successfully exploited for the detection of metastasis of most epithelial tumors by positron emission tomography combined with computed tomography (CT; PET/CT) [57, 58]. The Warburg effect seems to be achieved through stable genetic or epigenetic alterations that promote the constitutive activation of the glycolytic pathway and induce a decrease in mitochondrial oxidative phosphorylation, a phenomenon known as aerobic glycolysis. The transcription factor HIF $1 \alpha$ is one of the central players of cancer-specific aerobic glycolysis. Indeed, its stabilization leads to overexpression of target genes involved in key regulation steps of glucose transport, glycolysis, lactate production, and lactate/ proton extrusion [59]. Concomitantly, deregulated HIF $1 \alpha$ also induces suppression of mitochondrial metabolic pathways, such as oxidative phosphorylation, lipid synthesis, and $\beta$-oxidation [60].

The role of HIF $1 \alpha$ has been well documented in cancers originating in the ovary. Tumor xenografts obtained from stable HIF1 $\alpha$-silenced ovarian cancer cells show increased cell death and necrosis [61], and the expression levels of HIF $1 \alpha$ have been proposed as an independent prognostic factor in patients with epithelial ovarian tumors [62]. HIF1 $\alpha$ activity is regulated by several pathways, including the mitogen-activated protein kinase cascade, and p38 $\alpha$ has been demonstrated to be involved in the stabilization of HIF $1 \alpha$ in various normal and cancer cell types $[63,64]$. The p38 $\alpha$ pathway regulates proliferation, differentiation, metabolism, 
and cell death in a cell type-specific and signal-dependent manner [65]. Starting from our promising results obtained on colorectal cancer, showing that p $38 \alpha$ blockade promotes autophagy, cell cycle arrest, and non-apoptotic programmed cell death both in vitro and in vivo [66-69], we recently demonstrated that ovarian cancer cells are highly sensitive to $\mathrm{p} 38 \alpha$ inhibition [70]. Inhibition of p38 $\alpha$ activity by the specific inhibitor SB202190 impairs the expression of genes sustaining the altered metabolism of ovarian cancer cell lines and induces a shift from HIF $1 \alpha$ - to FoxO3A-dependent transcription (Figure 1) [70]. SB202190 promotes a timedependent reduction of HIF $1 \alpha$ protein levels, ultimately leading to an acute energy need that triggers the activation of AMPK and the consequent induction of the FoxO3A transcriptional program. In turn, FoxO3A promotes upregulation of crucial mediators of autophagy, cell cycle control, and cell death. Upon p38 $\alpha$ inhibition, autophagy is first accompanied by G1 arrest, but prolonged inactivation of p $38 \alpha$ leads to autophagic cell death [70]. Autophagy represents a promising target for the design of new therapeutic strategies relying on pharmacological manipulation in tumors displaying resistance to apoptosis. Besides, as aerobic glycolysis represents a differentiating factor between normal and cancer cells, inhibition of genes involved in cancer cell metabolic reprogramming may provide both specificity and efficacy in countering the energetic demand of transformed cells, thus hampering growth and inducing energy failure-dependent death processes. Thus, therapies based on p38 $\alpha$-specific inhibitors could represent a valuable tool against cancer.

The rationale to manipulate the $\mathrm{p} 38$ pathway in ovarian cancer is further corroborated by recent findings indicating that p38 $\alpha$ is a major mediator of drug resistance in response to chemotherapy with 5-fluorouracil and irinotecan $[71,72]$. Moreover, as p $38 \alpha$ inhibitory compounds are currently being investigated in clinical trials for inflammatory diseases and cancer [73], these findings might be taken advantage of in the prospect of clinical translation and support the idea that p38 $\alpha$ could be one of the special agents engaged by clinicians to hunt down the silent killer.

\section{Acknowledgments}

The authors thank Dr Francesco Paolo Jori for his helpful discussion during preparation of the paper and editorial assistance. Dr. Grossi is supported by a "Fondazione Negri Sud ONLUS" fellowship. This work was partially supported by a "My First Grant 2007" and an Investigator Grant 2010 (IG10177) (to C. Simone) from the Italian Association for Cancer Research (AIRC).

\section{References}

[1] T. A. Yap, C. P. Carden, and S. B. Kaye, "Beyond chemotherapy: targeted therapies in ovarian cancer," Nature Reviews Cancer, vol. 9, no. 3, pp. 167-181, 2009.

[2] J. S. Berek, "Epithelial ovarian cancer," in Practical Gynecologic Oncology 4th edn Ch. 11 Ovarian Cancer, J. S. Berek and N. F. Hacker, Eds., pp. 443-511, Lippincott Williams \& Wilkins, Philadelphia, Pa, USA, 2005.
[3] P. Watson and H. T. Lynch, "Extracolonic cancer in hereditary nonpolyposis colorectal cancer," Cancer, vol. 71, no. 3, pp. 677-685, 1993.

[4] S. C. Rubin, M. A. Blackwood, C. Bandera et al., "BRCA1, BRCA2, and hereditary nonpolyposis colorectal cancer gene mutations in an unselected ovarian cancer population: relationship to family history and implications for genetic testing," American Journal of Obstetrics and Gynecology, vol. 178, no. 4, pp. 670-677, 1998.

[5] G. Singer, R. Oldt III, Y. Cohen et al., "Mutations in BRAF and KRAS characterize the development of low-grade ovarian serous carcinoma," Journal of the National Cancer Institute, vol. 95, no. 6, pp. 484-486, 2003.

[6] K. Nakayama, N. Nakayama, R. J. Kurman et al., "Sequence mutations and amplification of PIK3CA and AKT2 genes in purified ovarian serous neoplasms," Cancer Biology and Therapy, vol. 5, no. 7, pp. 779-785, 2006.

[7] D. M. Dinulescu, T. A. Ince, B. J. Quade, S. A. Shafer, D. Crowley, and T. Jacks, "Role of K-ras and Pten in the development of mouse models of endometriosis and endometrioid ovarian cancer," Nature Medicine, vol. 11, no. 1, pp. 63-70, 2005.

[8] E. Oliva, E. F. Sarrió, E. F. Brachtel et al., "High frequency of $\beta$-catenin mutations in borderline endometrioid tumours of the ovary," Journal of Pathology, vol. 208, no. 5, pp. 708-713, 2006.

[9] R. Wu, N. Hendrix-Lucas, R. Kuick et al., "Mouse model of human ovarian endometrioid adenocarcinoma based on somatic defects in the Wnt/ $\beta$-catenin and PI3K/Pten signaling pathways," Cancer Cell, vol. 11, no. 4, pp. 321-333, 2007.

[10] K. W. Cheng, J. P. Lahad, J. W. Gray, and G. B. Mills, "Emerging role of RAB GTPases in cancer and human disease," Cancer Research, vol. 65, no. 7, pp. 2516-2519, 2005.

[11] I. Chefetz, J. C. Holmberg, A. B. Alvero, I. Visintin, and G. Mor, "Inhibition of Aurora-A kinase induces cell cycle arrest in epithelial ovarian cancer stem cells by affecting $\mathrm{NF} \kappa \mathrm{B}$ pathway," Cell Cycle, vol. 10, no. 13, pp. 2206-2214, 2011.

[12] S. Jones, T. L. Wang, I. M. Shih et al., "Frequent mutations of chromatin remodeling gene ARID1A in ovarian clear cell carcinoma," Science, vol. 330, no. 6001, pp. 228-231, 2010.

[13] K. C. Wiegand, S. P. Shah, O. M. Al-Agha et al., "ARID1A mutations in endometriosis-associated ovarian carcinomas," New England Journal of Medicine, vol. 363, no. 16, pp. 1532$1543,2010$.

[14] B. Guan, T.-L. Mao, P. K. Panuganti et al., "Mutation and loss of expression of ARID1A in uterine low-grade endometrioid carcinoma," American Journal of Surgical Pathology, vol. 35, no. 5, pp. 625-632, 2011.

[15] B. Guan, T.-L. Wang, and I.-M. Shih, "ARID1A, a factor that promotes formation of SWI/SNF-mediated chromatin remodeling, is a tumor suppressor in gynecologic cancers," Cancer Research, vol. 71, no. 21, pp. 6718-6727, 2011.

[16] Z. Lu, R. Z. Luo, Y. Lu et al., “The tumor suppressor gene ARHI regulates autophagy and tumor dormancy in human ovarian cancer cells," Journal of Clinical Investigation, vol. 118, no. 12, pp. 3917-3929, 2008.

[17] Y. Yu, F. Xu, H. Peng et al., "NOEY2 (ARHI), an imprinted putative tumor suppressor gene in ovarian and breast carcinomas," Proceedings of the National Academy of Sciences of the United States of America, vol. 96, no. 1, pp. 214-219, 1999.

[18] R. Z. Luo, H. Peng, F. Xu et al., "Genomic structure and promoter characterization of an imprinted tumor suppressor gene ARHI," Biochimica et Biophysica Acta, vol. 1519, no. 3, pp. 216-222, 2001. 
[19] D. G. Rosen, L. Wang, A. N. Jain et al., "Expression of the tumor suppressor gene ARHI in epithelial ovarian cancer is associated with increased expression of p21WAF1/CIP1 and prolonged progression-free survival," Clinical Cancer Research, vol. 10, no. 19, pp. 6559-6566, 2004.

[20] Y. Kondo, T. Kanzawa, R. Sawaya, and S. Kondo, "The role of autophagy in cancer development and response to therapy," Nature Reviews Cancer, vol. 5, no. 9, pp. 726-734, 2005.

[21] J. Marx, "Autophagy: is it cancer's friend or foe?" Science, vol. 312, no. 5777, pp. 1160-1161, 2006.

[22] K. Degenhardt, R. Mathew, B. Beaudoin et al., "Autophagy promotes tumor cell survival and restricts necrosis, inflammation, and tumorigenesis," Cancer Cell, vol. 10, no. 1, pp. 51-64, 2006.

[23] J. S. Carew, S. T. Nawrocki, C. N. Kahue et al., "Targeting autophagy augments the anticancer activity of the histone deacetylase inhibitor SAHAto overcome Bcr-Abl-mediated drug resistance," Blood, vol. 110, no. 1, pp. 313-322, 2007.

[24] Z. Lu, R. Z. Luo, Y. Lu et al., "A novel tumor suppressor gene ARHI induces autophagy and tumor dormancy in ovarian cancer xenografts," Journal of Clinical Investigation, vol. 118, pp. 3917-3929, 2008.

[25] W. Feng, R. T. Marquez, Z. Lu et al., "Imprinted tumor suppressor genes ARHI and PEG3 are the most frequently downregulated in human ovarian cancers by loss of heterozygosity and promoter methylation," Cancer, vol. 112, no. 7, pp. 14891502, 2008.

[26] B. Rosenberg, L. VanCamp, J. E. Trosko, and V. H. Mansour, "Platinum compounds: a new class of potent antitumour agents," Nature, vol. 222, no. 5191, pp. 385-386, 1969.

[27] C. W. Helm and J. C. States, "Enhancing the efficacy of cisplatin in ovarian cancer treatment-could arsenic have a role," Journal of Ovarian Research, vol. 2, no. 1, article 2, 2009.

[28] R. E. Bristow, R. S. Tomacruz, D. K. Armstrong, E. L. Trimble, and F. J. Montz, "Survival effect of maximal cytoreductive surgery for advanced ovarian carcinoma during the platinum era: a meta-analysis," Journal of Clinical Oncology, vol. 20, no. 5, pp. 1248-1259, 2002.

[29] R. F. Ozols, B. N. Bundy, B. E. Greer et al., "Phase III trial of carboplatin and paclitaxel compared with cisplatin and paclitaxel in patients with optimally resected stage III ovarian cancer: a Gynecologic Oncology Group study," Journal of Clinical Oncology, vol. 21, no. 17, pp. 3194-3200, 2003.

[30] W. P. McGuire, M. F. Brady, and R. F. Ozols, "The Gynecologic Oncology Group experience in ovarian cancer," Annals of Oncology, vol. 10, supplement 1, pp. S29-S34, 1999.

[31] J. Yoneda, H. Kuniyasu, M. A. Crispens, J. E. Price, C. D. Bucana, and I. J. Fidler, "Expression of angiogenesis-related genes and progression of human ovarian carcinomas in nude mice," Journal of the National Cancer Institute, vol. 90, no. 6, pp. 447-454, 1998.

[32] M. J. Birrer, M. E. Johnson, K. Hao et al., "Whole genome oligonucleotide-based array comparative genomic hybridization analysis identified fibroblast growth factor 1 as a prognostic marker for advanced-stage serous ovarian adenocarcinomas," Journal of Clinical Oncology, vol. 25, no. 16, pp. 22812287, 2007.

[33] B. J. Monk, E. Han, C. A. Josephs-Cowan, G. Pugmire, and R. A. Burger, "Salvage bevacizumab (rhuMAB VEGF)-based therapy after multiple prior cytoxic regimens in advanced refractory epithelial ovarian cancer," Gynecologic Oncology, vol. 102, pp. 140-144, 2006.

[34] A. A. Kamat, T. J. Kim, C. N. Landen et al., "Metronomic chemotherapy enhances the efficacy of antivascular therapy in ovarian cancer," Cancer Research, vol. 67, no. 1, pp. 281-288, 2007.

[35] C. Lu, P. H. Thaker, Y. G. Lin et al., "Impact of vessel maturation on antiangiogenic therapy in ovarian cancer," American Journal of Obstetrics and Gynecology, vol. 198, no. 4, pp. 477-479, 2008.

[36] J. J. Biagi, A. M. Oza, H. I. ChalChal et al., "A phase II study of sunitinib in patients with recurrent epithelial ovarian and primary peritoneal carcinoma: an NCIC clinical trials group study," Annals of Oncology, vol. 22, no. 2, pp. 335-340, 2011.

[37] H. W. Hirte, L. Vidal, and G. F. Fleming, "Aphase II study of cediranib (AZD2171) in recurrent or persistent ovarian, peritonealor fallopian tube cancer: final results of aPMH, Chicago and California consortiatrial," in Proceedings of the 44th American Society of Clinical OncologyAnnual Meeting of the Program and Abstracts, Chicago, Ill, USA, May-June 2008.

[38] R. J. Schilder, M. W. Sill, X. Chen et al., "Phase II study of gefitinib inpatients with relapsed or persistent ovarian or primaryperitoneal carcinoma and evaluation of epidermalgrowth factor receptor mutations andimmunohistochemical expression: a Gynecologic Oncology Group Study," Clinical Cancer Research, vol. 11, pp. 5539-5548, 2005.

[39] A. N. Gordon, N. Finkler, R. P. Edwards et al., "Efficacy and safety of erlotinib $\mathrm{HCl}$, an epidermal growth factor receptor (HER1/EGFR) tyrosine kinase inhibitor, in patients with advanced ovarian carcinoma: results from a phase II multicenter study," International Journal of Gynecological Cancer, vol. 15, no. 5, pp. 785-792, 2005.

[40] V. Heinemann, S. Stintzing, T. Kirchner, S. Boeck, and A. Jung, "Clinical relevance of EGFR- and KRAS-status in colorectal cancer patients treated with monoclonal antibodies directed against the EGFR," Cancer Treatment Reviews, vol. 35, no. 3, pp. 262-271, 2009.

[41] H. S. Cho, K. Mason, K. X. Ramyar et al., "Structure of the extracellular region of HER2 alone and in complex with the Herceptin Fab," Nature, vol. 421, no. 6924, pp. 756-760, 2003.

[42] M. C. Franklin, K. D. Carey, F. F. Vajdos, D. J. Leahy, A. M. De Vos, and M. X. Sliwkowski, "Insights into ErbB signaling from the structure of the ErbB2-pertuzumab complex," Cancer Cell, vol. 5, no. 4, pp. 317-328, 2004.

[43] S. Makhija, L. C. Amler, D. Glenn et al., "Clinical activity of gemcitabine plus pertuzumab in platinum-resistant ovarian cancer, fallopian tube cancer, or primary peritoneal cancer," Journal of Clinical Oncology, vol. 28, no. 7, pp. 1215-1223, 2010.

[44] S. B. Kaye, C. J. Poole, and M. Bidzinksi, "A randomised phase II study evaluating the combination of carboplatin-based chemotherapy with pertuzumab versus carboplatin based therapy alone in patients with relapsed, platinum sensitive ovarian cancer," Journal of Clinical Oncology, vol. 26, abstract 5520, 2008.

[45] D. Faratian, A. J.M. Zweemer, Y. Nagumo et al., "Trastuzumab and pertuzumab produce changes in morphology and estrogen receptor signaling in ovarian cancer xenografts revealing new treatment strategies," Clinical Cancer Research, vol. 17, no. 13, pp. 4451-4461, 2011.

[46] L. Hu, J. Hofmann, Y. Lu, G. B. Mills, and R. B. Jaffe, "Inhibition of phosphatidylinositol 3'-kinase increases efficacy of paclitaxel in in vitro and in vivo ovarian cancer models," Cancer Research, vol. 62, no. 4, pp. 1087-1092, 2002.

[47] F. I. Raynaud, S. Eccles, P. A. Clarke et al., "Pharmacologic characterization of a potent inhibitor of class I phosphatidylinositide 3-kinases," Cancer Research, vol. 67, no. 12, pp. 58405850, 2007. 
[48] J. S. McMurray, "A new small-molecule Stat3 inhibitor," Chemistry and Biology, vol. 13, no. 11, pp. 1123-1124, 2006.

[49] H. P. Beck, T. Kohn, S. Rubenstein et al., "Discovery of potent $\mathrm{LPA}_{2}$ (EDG4) antagonists as potential anticancer agents," Bioorganic and Medicinal Chemistry Letters, vol. 18, no. 3, pp. 1037-1041, 2008.

[50] Y. G. Lin, A. B. Kunnumakkara, A. Nair et al., "Curcumin inhibits tumor growth and angiogenesis in ovarian carcinoma by targeting the nuclear factor- $\kappa \mathrm{B}$ pathway," Clinical Cancer Research, vol. 13, no. 11, pp. 3423-3430, 2007.

[51] A. K. Samanta, H. J. Huang, R. C. Bast, and W. S. L. Liao, "Overexpression of $\mathrm{MEKK}_{3}$ confers resistance to apoptosis through activation of NF $\kappa \mathrm{B}$," Journal of Biological Chemistry, vol. 279, no. 9, pp. 7576-7583, 2004.

[52] M. Karin, "Nuclear factor- $\kappa \mathrm{B}$ in cancer development and progression," Nature, vol. 441, no. 7092, pp. 431-436, 2006.

[53] A. Ashworth, "A synthetic lethal therapeutic approach: pol$y(A D P)$ ribose polymerase inhibitors for the treatment of cancers deficient in DNA double-strand break repair," Journal of Clinical Oncology, vol. 26, no. 22, pp. 3785-3790, 2008.

[54] P. C. Fong, D. S. Boss, and C. P. Carden, "AZD2281 (KU0059436), a PARP (poly ADP-ribose polymerase) inhibitor with single agent anticancer activity in patients with BRCA deficient ovarian cancer: results from a phase I study," Journal of Clinical Oncology, vol. 26, abstract 5510, 2008.

[55] A. Mangerich and A. Bürkle, "How to kill tumor cells with inhibitors of poly(ADP-ribosyl)ation," International Journal of Cancer, vol. 128, no. 2, pp. 251-265, 2011.

[56] N. C. Denko, "Hypoxia, HIF1 and glucose metabolism in the solid tumour," Nature Reviews Cancer, vol. 8, no. 9, pp. 705713, 2008 .

[57] S. S. Gambhir, "Molecular imaging of cancer with positron emission tomography," Nature Reviews Cancer, vol. 2, no. 9, pp. 683-693, 2002.

[58] D. A. Mankoff, J. F. Eary, J. M. Link et al., "Tumor-specific positron emission tomography imaging in patients: $\left[{ }^{18} \mathrm{~F}\right]$ fluorodeoxyglucose and beyond," Clinical Cancer Research, vol. 13, no. 12 , pp. 3460-3469, 2007.

[59] G. Kroemer and J. Pouyssegur, "Tumor cell metabolism: cancer's achilles' heel," Cancer Cell, vol. 13, no. 6, pp. 472-482, 2008.

[60] P. P. Hsu and D. M. Sabatini, "Cancer cell metabolism: warburg and beyond," Cell, vol. 134, no. 5, pp. 703-707, 2008.

[61] E. Favaro, G. Nardo, L. Persano et al., "Hypoxia inducible factor- $1 \alpha$ inactivation unveils a link between tumor cell metabolism and hypoxia-induced cell death," American Journal of Pathology, vol. 173, no. 4, pp. 1186-1201, 2008.

[62] R. Shimogai, J. Kigawa, H. Itamochi et al., "Expression of hypoxia-inducible factor $1 \alpha$ gene affects the outcome in patients with ovarian cancer," International Journal of Gynecological Cancer, vol. 18, no. 3, pp. 499-505, 2008.

[63] B. M. Emerling, L. C. Platanias, E. Black, A. R. Nebreda, R. J. Davis, and N. S. Chandel, "Mitochondrial reactive oxygen species activation of p38 mitogen-activated protein kinase is required for hypoxia signaling," Molecular and Cellular Biolo$g y$, vol. 25, no. 12, pp. 4853-4862, 2005.

[64] S. J. Kwon, J. J. Song, and Y. J. Lee, "Signal pathway of hypoxia-inducible factor- $1 \alpha$ phosphorylation and its interaction with von Hippel-Lindau tumor suppressor protein during ischemia in MiaPaCa-2 pancreatic cancer cells," Clinical Cancer Research, vol. 11, no. 21, pp. 7607-7613, 2005.

[65] F. Chiacchiera and C. Simone, "Signal-dependent regulation of gene expression as a target for cancer treatment: inhibiting p38 $\alpha$ in colorectal tumors," Cancer Letters, vol. 265, no. 1, pp. 16-26, 2008.

[66] F. Comes, A. Matrone, P. Lastella et al., "A novel cell typespecific role of $\mathrm{p} 38 \alpha$ in the control of autophagy and cell death in colorectal cancer cells," Cell Death and Differentiation, vol. 14, no. 4, pp. 693-702, 2007.

[67] C. Simone, "Signal-dependent control of autophagy and cell death in colorectal cancer cell: the role of the p38 pathway," Autophagy, vol. 3, no. 5, pp. 468-471, 2007.

[68] F. Chiacchiera, A. Matrone, E. Ferrari et al., "p38 $\alpha$ blockade inhibits colorectal cancer growth in vivo by inducing a switch from HIF $1 \alpha$ - to FoxO-dependent transcription," Cell Death and Differentiation, vol. 16, no. 9, pp. 1203-1214, 2009.

[69] F. Chiacchiera and C. Simone, "Inhibition of p38 $\alpha$ unveils an AMPK-FoxO3A axis linking autophagy to cancer-specific metabolism," Autophagy, vol. 5, no. 7, pp. 1030-1033, 2009.

[70] A. Matrone, V. Grossi, F. Chiacchiera et al., "P38 $\alpha$ is required for ovarian cancer cell metabolism and survival," International Journal of Gynecological Cancer, vol. 20, no. 2, pp. 203-211, 2010.

[71] S. Y. Yang, A. Miah, K. M. Sales, B. Fuller, A. M. Seifalian, and M. Winslet, "Inhibition of the p38 MAPK pathway sensitises human colon cancer cells to 5-fluorouracil treatment," International Journal of Oncology, vol. 38, no. 6, pp. 1695-1702, 2011.

[72] S. Paillas, F. Boissière, F. Bibeau et al., "Targeting the p38 MAPK pathway inhibits irinotecan resistance in colon adenocarcinoma," Cancer Research, vol. 71, no. 3, pp. 1041-1049, 2011.

[73] E. F. Wagner and A. R. Nebreda, "Signal integration by JNK and p38 MAPK pathways in cancer development," Nature Reviews Cancer, vol. 9, no. 8, pp. 537-549, 2009. 


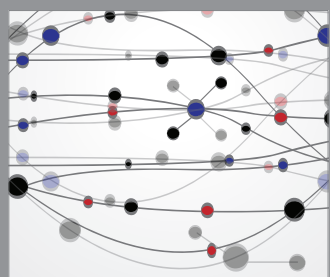

The Scientific World Journal
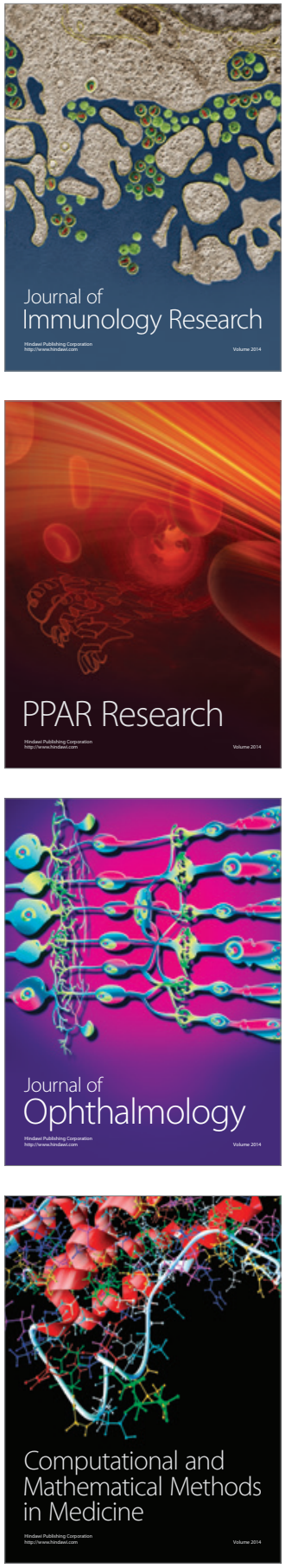

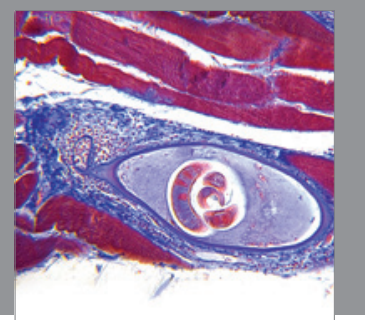

Gastroenterology

Research and Practice
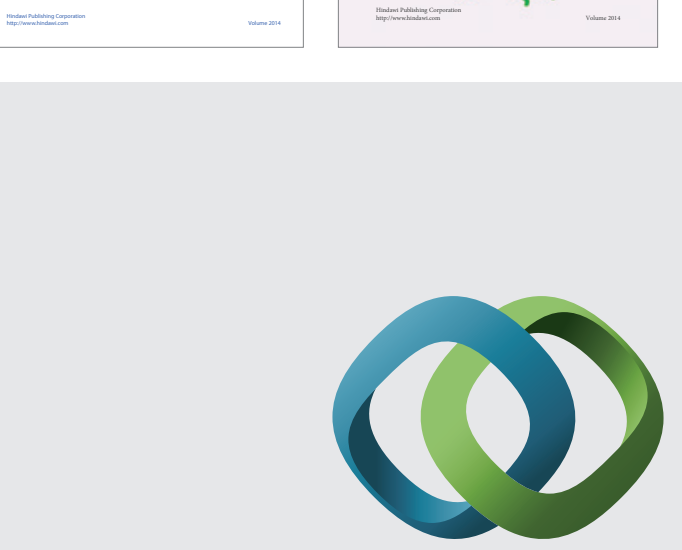

\section{Hindawi}

Submit your manuscripts at

http://www.hindawi.com
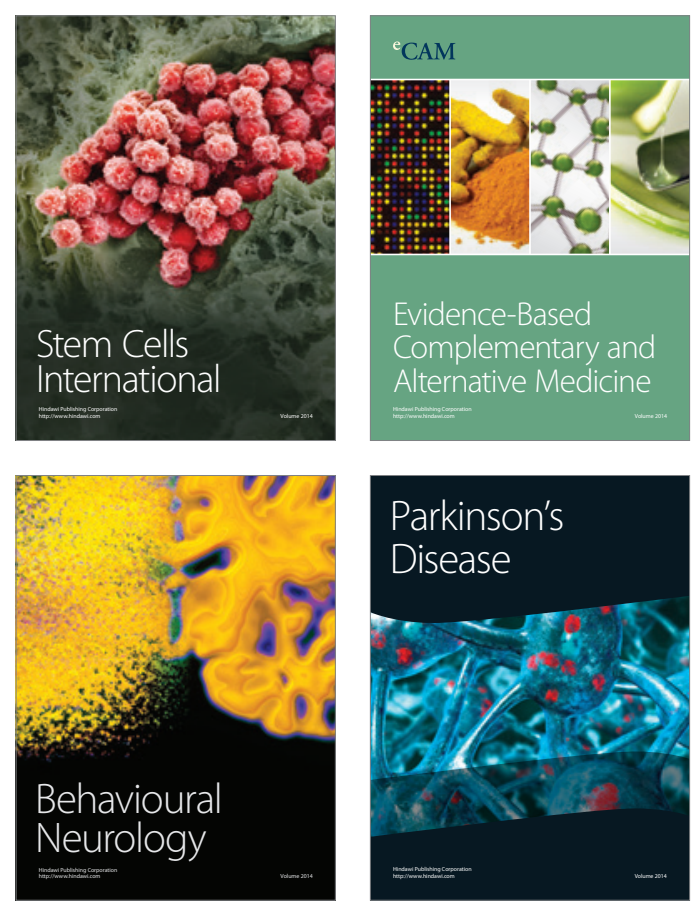

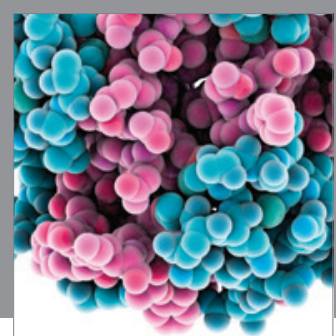

Journal of
Diabetes Research

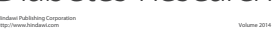

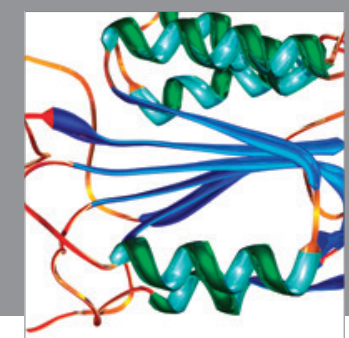

Disease Markers
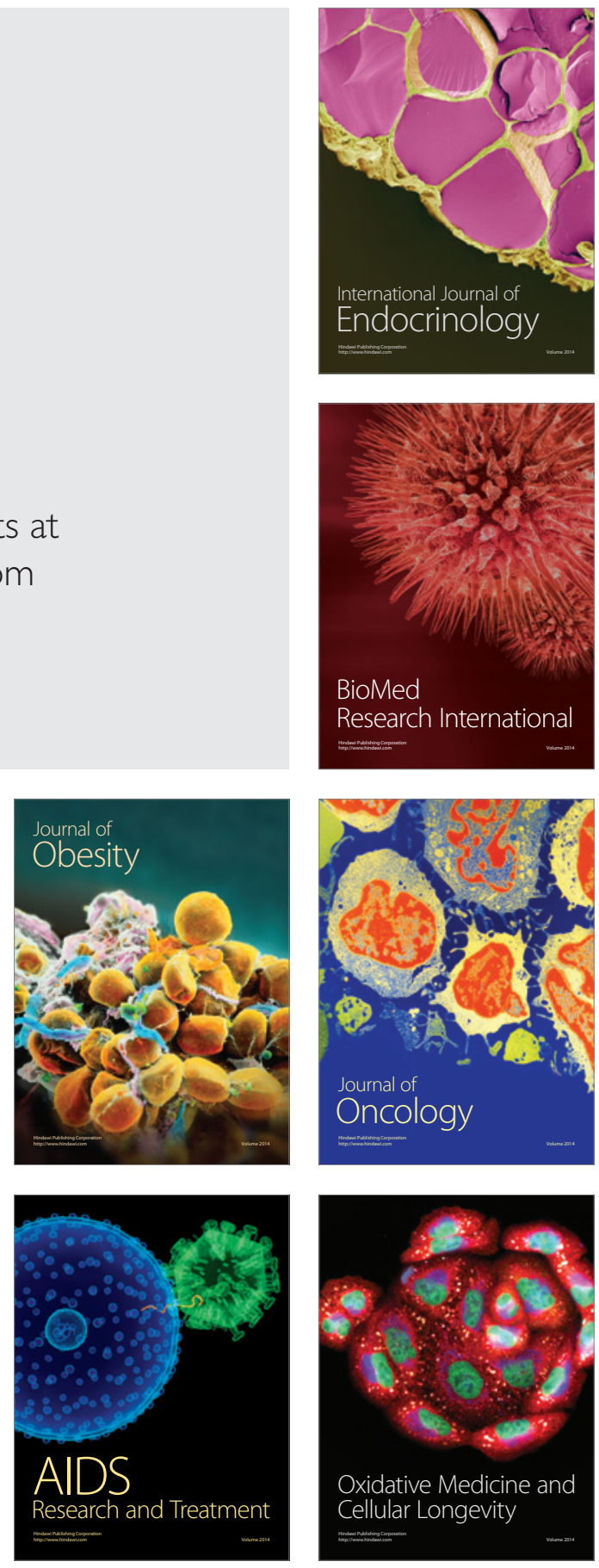